\title{
Towards a classification of wave catastrophes
}

\author{
T Kiss ${ }^{1,2}$ and U Leonhardt ${ }^{3}$ \\ ${ }^{1}$ Research Institute for Solid State Physics and Optics, H-1525 Budapest, PO Box 49, \\ Hungary \\ ${ }^{2}$ Institute of Physics, University of Pécs, Ifjúság u. 6, H-7624 Pécs, Hungary \\ ${ }^{3}$ School of Physics and Astronomy, University of St Andrews, North Haugh, St Andrews \\ KY16 9SS, UK
}

Received 1 September 2003, accepted for publication 23 December 2003 Published 19 April 2004

Online at stacks.iop.org/JOptA/6/S246

DOI: $10.1088 / 1464-4258 / 6 / 5 / 019$

\begin{abstract}
Wave catastrophes are characterized by logarithmic phase singularities. Examples are light at the horizon of a black hole, sound in transsonic fluids, waves in accelerated frames, light in singular dielectrics and slow light close to a zero of the group velocity. We show that the wave amplitude grows with a half-integer power for monodirectional and symmetric wave catastrophes.
\end{abstract}

Keywords: waves at horizons, logarithmic phase singularities

Imagine light propagating away from a black hole. Suppose that the light has been emitted immediately before the horizon. A distant observer decomposes the light into its spectral components with frequencies $\omega$. The wavelength of each spectral component must shrink to zero close to the horizon, in order to compensate for the infinite gravitational red-shift here. It turns out [1] that the wavelength is proportional to the radial distance from the horizon. Consequently, the wavenumber depends inversely on the distance and the phase diverges logarithmically. Such rapid oscillations occur only in the direction orthogonal to the horizon, which allows us to ignore all other spatial dimensions and to focus on an effectively $(1+1)$-dimensional model where we can also ignore the polarization of light. In this model a monochromatic wave $\varphi$ behaves like

$$
\varphi \sim\left(z-z_{0}\right)^{\mathrm{i} v} \mathrm{e}^{-\mathrm{i} \omega t}
$$

near the horizon at $z_{0}$ where $z$ denotes the spatial coordinate and $t$ the time. Note that the behaviour (1) may extend also beyond the horizon for negative $z-z_{0}$ [1]. There are two independent ways of connecting waves at positive and at negative $z-z_{0}$, on the upper or the lower half of the complex $z$ plane, generating two different amplitudes. This extra degree of freedom indicates that the horizon cuts space into two disconnected regions. The dimensionless power iv characterizes the wave singularity at the horizon. The real part of $v$ gives the number of phase cycles per e-fold of $z-z_{0}$ and the imaginary part of $v$ describes how the amplitude grows near the horizon. For a black hole $v$ equals $\omega / \alpha$ where $\alpha$ denotes the gravitational acceleration at the horizon divided by the speed of light.

Logarithmic phase singularities of the type (1) are not confined to the physics of waves near black holes. Sound waves in fluids suffer a similar fate in transsonic flows (at sonic horizons) [2-5] and so do waves in accelerated frames (Rindler coordinates) [1], light in singular dielectrics [6] and slow light close to a zero of the group velocity $[7,8]$. We refer to a behaviour of the type (1) as a wave catastrophe [7, 8], because the horizon fundamentally alters the causal structure of wave propagation. Many wave catastrophes are caused by catastrophic events such as the gravitational collapse or breaking the sound barrier. Furthermore, the simple laws of wave propagation that lead to the characteristic behaviour (1) must break down when the wavelength shrinks beyond all scales. The theory predicts its own demise - a theoretical catastrophe $[9,10]$.

Note that the wave catastrophes do not belong to the mathematical subject of catastrophe theory $[11,12]$ with its known classification of catastrophes. The wave catastrophes do not involve singularities of gradient maps [11, 12] like the singularities of light rays in diffraction catastrophes [13, 14]. However, the two theoretical concepts share a similar spirit: in diffraction catastrophes [13, 14] the rules of geometric optics break down and, similarly, wave catastrophes are superseded by small-scale effects. More importantly, in diffraction catastrophes $[13,14]$ the ray catastrophes are accompanied by characteristic wave effects, i.e. interference patterns, and the wave catastrophes may be responsible for characteristic 
Towards a classification of wave catastrophes

quantum effects $[7,8,15,16]$, in particular spontaneous particle production $[1,17]$.

The spectrum of the generated quantum radiation depends on the imaginary part of the index $v[7,8]$ and it seems to be independent of the small-scale physics where the theory (1) is not applicable any longer $[5,18,19]$. All the examples of wave catastrophes studied so far correspond to

$$
\operatorname{Im} v=\frac{n}{2}, \quad n \in Z .
$$

In this paper we develop a simple argument showing that the property (2) is not a coincidence for two general classes of wave catastrophes:

(A) The catastrophe affects only waves propagating in one direction, such as the outgoing waves from the horizon of a black hole where incident waves are not singular. In this case only one non-zero $v$ exists at the horizon $z_{0}$.

(B) The catastrophe affects both directions equally. This case corresponds to two powers iv that are complex conjugate, one describing waves propagating to the right and the other referring to waves propagating to the left.

Waves in accelerated frames [1], light in singular dielectrics [6] and slow-light catastrophes $[7,8]$ belong to case (B).

Consider real scalar waves $\phi$ in $(1+1)$-dimensions that are subject to the principle of least action. We assume that the wave equation is linear and of second order. Consequently the most general Lagrangian for $\phi$ is

$$
\mathscr{L}=A^{\mu \mu^{\prime}}\left(\partial_{\mu} \phi\right)\left(\partial_{\mu^{\prime}} \phi\right)+2 B^{\mu} \phi \partial_{\mu} \phi+C \phi^{2} .
$$

The indices refer to the time $t(\mu=0)$ and to the spatial coordinate $z(\mu=1)$, the $\partial_{\mu}$ denote partial derivatives and we employ Einstein's summation convention. Without loss of generality we assume that

$$
A^{\mu \mu^{\prime}}=A^{\mu^{\prime} \mu}
$$

We express the Lagrangian as

$$
\mathscr{L}=A^{\mu \mu^{\prime}}\left(\partial_{\mu} \phi\right)\left(\partial_{\mu^{\prime}} \phi\right)+\phi^{2}\left(C-\partial_{\mu} B^{\mu}\right)+\partial_{\mu}\left(B^{\mu} \phi^{2}\right) .
$$

Since the divergence $\partial_{\mu}\left(B^{\mu} \phi^{2}\right)$ does not influence the action and hence the equations of motion, we can reduce the problem to a Lagrangian of the form

$$
\mathscr{L}=A^{\mu \mu^{\prime}}\left(\partial_{\mu} \phi\right)\left(\partial_{\mu^{\prime}} \phi\right)-M \phi^{2} .
$$

We obtain the Euler-Lagrange equations

$$
\partial_{\mu} A^{\mu \mu^{\prime}} \partial_{\mu^{\prime}} \phi+M \phi=0 .
$$

We assume a stationary regime where $A^{\mu \mu^{\prime}}$ and $M$ may depend on $z$, but not on $t$, and we decompose $\phi$ into monochromatic waves $\varphi$ with

$$
\partial_{t} \varphi=-\mathrm{i} \omega \varphi \text {. }
$$

We get

$$
\left(-\omega^{2} A^{00}-2 \mathrm{i} \omega A^{01} \partial_{z}-\mathrm{i} \omega\left(\partial_{z} A^{01}\right)+\partial_{z} A^{11} \partial_{z}+M\right) \varphi=0 .
$$

In order to obtain a solution of the type (1) near $z_{0}$, the $A^{\mu \mu}$ matrix and $M$ must behave like

$$
\begin{gathered}
A^{\mu \mu^{\prime}} \sim \gamma\left(\begin{array}{cc}
\frac{\alpha}{z-z_{0}} & \beta \\
\beta & z_{0}-z
\end{array}\right)\left(z-z_{0}\right)^{n}, \\
M \sim \gamma \delta\left(z-z_{0}\right)^{n-1}
\end{gathered}
$$

with the $\alpha, \beta, \gamma, \delta$ constants. The power $n$ must be integer, because otherwise the Lagrangian (6) is not real for all $\phi$. We substitute the structure (10) into the wave equation (9) and solve for $v$ with the result

$$
v=i \frac{n}{2}-\beta \omega \pm \sqrt{\left(\alpha+\beta^{2}\right) \omega^{2}-\delta^{2}-\frac{n^{2}}{4}} .
$$

If the wave catastrophe affects only waves propagating in one direction, like the outgoing waves from a black hole, one of the $v$ is zero. Consequently, the other is in $/ 2-2 \beta \omega$. In the case where the catastrophe affects both directions equally, $\beta$ must vanish. If $v$ corresponds to a wave catastrophe it must have a non-vanishing real part. Consequently, the imaginary part of $v$ is $n / 2$.

This proves our statement. At wave catastrophes, i.e. at logarithmic phase singularities, the wave amplitudes rise with the power $n / 2$ for monodirectional and for symmetric catastrophes.

\section{Acknowledgments}

Our paper was supported by the ESF Programme Cosmology in the Laboratory, the Leverhulme Trust, the Hungarian Scientific Research Fund (contract No T43287), the Hungarian Academy of Sciences (Bolyai Ösztöndíj), and the Marie Curie Programme of the European Commission.

\section{References}

[1] Brout R, Massar S, Parentani R and Spindel Ph 1995 Phys. Rep. 260329

[2] Unruh W G 1981 Phys. Rev. Lett. 461351

[3] Visser M 1998 Class. Quantum Grav. 151767

[4] Garay L J, Anglin J R, Cirac J I and Zoller P 2000 Phys. Rev. Lett. 854643

[5] Leonhardt U, Kiss T and Öhberg P 2003 J. Opt. B: Quantum Semiclass. Opt. $5 \mathrm{~S} 42$

[6] Reznik B 2000 Phys. Rev. D 62044044

[7] Leonhardt U 2002 Nature 415406

[8] Leonhardt U 2002 Phys. Rev. A 65043818

[9] t'Hooft G 1985 Nucl. Phys. B 256727

[10] Jacobson T 1991 Phys. Rev. D 441731

[11] Thom R 1972 Stabilité Structurelle et Morphogénèse (Reading, MA: Benjamin)

Thom R 1975 Structural Stability and Morphogenesis (Reading, MA: Benjamin) (Engl. Transl.)

[12] Arnol'd V I 1975 Usp. Mat. Nauk 303 Arnol'd V I 1975 Russ. Math. Surv. 301 (Engl. Transl.)

[13] Berry M V and Upstill C 1980 Prog. Opt. 28257

[14] Nye J F 1999 Natural Focusing and Fine Structure of Light (Bristol: Institute of Physics Publishing)

[15] Berry M V 1992 Rays, wavefronts and phase: a picture book of cusps Huygen' Principle 1690-1990: Theory and Applications ed H Blok, H A Frewerda and H K Kuiken (Amsterdam: Elsevier)

[16] Berry M V 1998 Proc. SPIE 34871

[17] Hawking S M 1974 Nature 24830

[18] Unruh W G 1995 Phys. Rev. D 512827

[19] Brout R, Massar S, Parentani R and Spindel Ph 1995 Phys. Rev. D 524559 